\title{
Influence of spin-rotation measurements on partial-wave analyses of elastic pion-nucleon scattering
}

I. G. Alekseev, V. P. Kanavets, B. V. Morozov, D. N. Svirida

Institute for Theoretical and Experimental Physics

B. Cheremushkinskaya 25, 117259 Moscow, Russia

S. P. Kruglov, A. A. Kulbardis, V. V. Sumachev

Petersburg Nuclear Physics Institute

Gatchina, Leningrad district, 188350 Russia

R. A. Arndt, I. I. Strakovsky ${ }^{\dagger}$, and R. L. Workman

Department of Physics, Virginia Tech, Blacksburg, VA 24061

\begin{abstract}
Recent measurements of spin-rotation parameters in elastic $\pi^{+} p$ scattering are in marked disagreement with predictions of the Carnegie-Mellon-Berkeley and Karlsruhe-Helsinki analyses. Using the method of Barrelet, we show how this discrepancy can be removed. We then show how this Barrelet transformation alters the partial-wave amplitudes. The effect of unitarity and analyticity constraints is also considered.
\end{abstract}

PACS Numbers: 14.20.Gk, 13.30.Eg, 13.75.Gx, 11.80.Et 


\section{Introduction}

The excited states of the nucleon have been studied mainly through partial-wave analyses of $\pi N$ total, elastic and charge-exchange scattering data. The Review of Particle Properties [1] lists about 20 such states below $2 \mathrm{GeV}$. Many of these resonances have a 3-star or lower rating, which implies their existence is at most "likely" and their properties are uncertain. Most of these states were either found or verified in the Karlsruhe-Helsinki(KH) [2] and Carnegie-Mellon-Berkeley(CMB) [3] analyses.

In this paper we consider the effect of spin-rotation(SR) measurements on partial-wave analyses. In particular, we focus on the $\mathrm{KH}$ and CMB analyses, as no SR data were available in the resonance region when these analyses were performed. Measurements of $R$ and $A$ between $300 \mathrm{MeV}$ and $600 \mathrm{MeV}$ were recently made at LAMPF [4] and PNPI [5]. These quantities were at least qualitatively predicted by the existing analyses. This was not so surprising. Earlier studies [6] had suggested that the imposition of sufficient unitarity and analyticity constraints would remove those ambiguities due to the absence of SR data. However, more recent ITEP-PNPI measurements [7] of $A$ for $\pi^{+} p$ at $1.3 \mathrm{GeV}$ are surprising. These data, taken between 120 and 140 degrees, suggest an angular dependence very different from the $\mathrm{KH}$ and $\mathrm{CMB}$ predictions.

In Section II, we briefly review the problems that arise when analyses use incomplete sets of data (no spin-rotation measurements). The method of Barrelet [8] is used to isolate and modify one zero-trajectory which may be responsible for the poor fit to the $1.3 \mathrm{GeV}$ SR data. The effect of a transformed trajectory on the unitarity and analyticity of the $\mathrm{KH}$ and CMB solutions is also considered. In Section III, we show that the above procedure produces an improved agreement between the SR data and the $\mathrm{KH}$ analysis. Here we also compare the partial-wave amplitudes of the original and modified $\mathrm{KH}$ solution. Finally, in Section IV, we summarize our results and suggest extensions of the present work. 


\section{Amplitude Ambiguities and the Barrelet Method}

The $\mathrm{KH}$ and $\mathrm{CMB}$ analyses were performed prior to the existence of spin-rotation measurements in the resonance region. These analyses had cross section (differential and total) and polarization data. However, without further theoretical input, it is clear that this dataset is insufficient. This is easier to see if we work with transversity amplitudes $\left(F^{ \pm}=F \pm i G\right)$ constructed from the spin flip $(G)$ and spin non-flip $(F)$ amplitudes. In this representation, the differential cross section $(d \sigma / d \Omega)$ and polarization $(P)$

$$
\begin{aligned}
\frac{d \sigma}{d \Omega} & =\left|F^{+}\right|^{2}+\left|F^{-}\right|^{2}, \\
P \frac{d \sigma}{d \Omega} & =\left|F^{+}\right|^{2}-\left|F^{-}\right|^{2},
\end{aligned}
$$

determine $\left|F^{ \pm}\right|$, leaving an undetermined relative phase. (In addition to the relative phase between transversity amplitudes, there is also an undetermined overall phase [9].) The total cross sections further constrain the forward scattering amplitudes.

The symmetry $F^{+}(-\theta)=F^{-}(\theta)$ can be used to express the two transversity amplitudes in terms of a single function. Barrelet [8] showed that it was useful to parameterize this function as a product

$$
F(w)=\frac{F(1)}{w^{N}} \prod_{i=1}^{2 N} \frac{w-w_{i}}{1-w_{i}},
$$

in terms of the variable $w=e^{i \theta}$. The unit circle, $|w|=1$, corresponds to the physical region where $\theta$ is real and equal to the center-of-mass scattering angle.

In writing Eq.(3), we have implicitly assumed that $F(w)$ can be represented by a finite polynomial. The use of this form, in analyzing scattering data directly, has been criticized by Höhler [9]. He notes that the above product should contain another factor, $\Re(w)$, which accounts for both the distant zeros and the effects of branch cuts and poles which are known to exist but cannot be described by a polynomial. The violation of unitarity can also be a problem.

In the present work, we have applied the Barrelet method to the KA84 [2] solution. (Results for the CMB [3] and KH80 [2] solutions are similar.) Since the KH and CMB 
analyses have employed unitarity and analyticity constraints, we expect that they can be represented by the product given in Eq.(3), with the additional factor suggested in Ref. [9].

The operation $w_{i} \rightarrow 1 / w_{i}^{*}$ for a single term in the above product preserves both the cross section and polarization. This represents an ambiguity that can be resolved by SR measurements. When a zero trajectory crosses the unit circle, $w_{i}$ and $1 / w_{i}^{*}$ are equal. At this point, an alternate zero trajectory (with $w_{i} \rightarrow 1 / w_{i}^{*}$ ) can emerge. Whether this new trajectory connects reasonably smoothly to the original one also depends on the angle at which the trajectory crosses the unit circle.

We have identified one particular trajectory which can be linked to the discrepancy found in the SR measurements. This trajectory crosses the unit circle at about $700 \mathrm{MeV}$ and remains influential through the remainder of the resonance region. When transformed, it produces an improved description of the SR data. This trajectory appears in both the $\mathrm{KH}$ and $\mathrm{CMB}$ solutions. The original and transformed trajectories for the KA84 solution are displayed in Fig. 1. A detailed comparison of the original and transformed solutions is given in Section III.

If SR data had become available at the time of the $\mathrm{KH}$ and $\mathrm{CMB}$ analyses, they would have been fitted along with the constraints from dispersion relations. We have found that the Barrelet transformation, applied to these solutions, results in a good fit to SR measurements without altering the fit to the remaining database. If this operation has a minimal effect on the dispersion relation constraints, we can take the variation in partial-wave amplitudes, displayed in Section III, as a guide to the results expected in a full partial-wave analysis.

As the transformation of roots does not alter the forward amplitude, none of the forward dispersion-relation integrals are affected. Gauging the effect on other dispersion relations is more difficult, since we have only the $A$ measurement for elastic $\pi^{+} p$ scattering $(\mathrm{I}=3 / 2)$. A change in the $I=3 / 2$ partial-waves would result in a readjustment of the $I=1 / 2$ amplitudes in a fit to the full database. As a simple test, we recalculated the $\pi N N$ coupling constant $\left(f^{2}\right)$ using the $\pi^{+} p$ amplitudes in a fixed-t dispersion relation. The value of $f^{2}$ was shifted systematically by less than $1 \%$ over a range of t-values. While this change in $f^{2}$ is within 
its uncertainty, some small readjustment of the amplitudes, due to the dispersion-relation constraints, would occur in a full analysis [10].

Finally, we should note that the Barrelet transformation can result in a violation of unitarity. This can be a serious problem if data are analyzed directly. Here we are again helped by the fact that we start with amplitudes which are unitary. The transformed amplitudes have been checked for violations of unitarity, and one example is given in the next section.

\section{Partial-Wave Amplitudes and Observables}

As mentioned in Section II, the Barrelet transformation leaves invariant the polarization and the differential and total cross sections. In Fig. 2, we show how much better the transformed amplitudes describe the $\pi^{+} p A$ measurements at $1.3 \mathrm{GeV}$. This change in the prediction for $A$ starts at about $700 \mathrm{MeV}$ and persists through the resonance region. The other sets of $R$ and $A$ measurements just missed this effect, as they extended up to only 600 $\mathrm{MeV}$. A contour plot of differences between the predictions of the original and transformed solutions is displayed in Fig. 3. This transformation also removes disagreements with the analysis of Ref. [11] at back angles. The KH and CMB solutions have resolved ambiguities in the same way [12] and, therefore, Figs. 2 and 3 are qualitatively the same when the CMB solution is used 13 .

Differences between the original and transformed KH amplitudes are shown in Fig. 4. Partial-waves with clear resonance signatures show the same qualitative behavior in both solutions. Weaker resonance signals are more significantly affected in the S, P and D waves. The higher partial waves are again qualitatively similar in the two solutions.

The $\mathrm{D}_{35}$ partial wave provides an example of a case where the Barrelet transformation results in a small violation of unitarity. (The imaginary part is negative.) Luckily, this problem occurs at energies where the imaginary part is very small.

\section{Conclusions}

We have shown that the Barrelet transformation of one particular zero trajectory has the

effect of greatly improving the $\mathrm{KH}$ and $\mathrm{CMB}$ descriptions of recent spin-rotation measure- 
ments. The effect of this transformation on the partial-wave amplitudes serves as a guide to results which would be expected in a full analysis. A further readjustment to satisfy unitarity and analyticity constraints should also be expected. This is particularly true for the $\mathrm{D}_{35}$ partial wave.

This transformation, applied to the $\mathrm{KH}$ and $\mathrm{CMB}$ solutions, also results in an improved agreement between the KH, CMB and VPI [11] predictions for $A$ in $\pi^{+} p$ scattering at back angles, over a wide range of energies. It is important to have further measurements in the neighborhood of $1.3 \mathrm{GeV}$ to determine whether the modified solutions make improved predictions.

There are numerous resonance candidates with masses near the center-of-mass energy of the ITEP-PNPI measurements [7]. From Fig. 4, we see that the well-established $\mathrm{F}_{35}(1905)$ and $\mathrm{F}_{37}(1950)$ resonances appear clearly in both the original and transformed solutions. Weaker structures in the S, P and D waves have been altered significantly in the transformed solution. This added uncertainty should be factored into future comparisons with quarkmodel predictions.

This work was supported in part by the U.S. Department of Energy Grant DE-FG0588ER40454 and the Russian Fund of Fundamental Research and Russian State ScientificTechnical Program "Fundamental Nuclear Physics". I. S. acknowledges the hospitality extended by the Physics Department of Virginia Tech. 


\section{REFERENCES}

$\dagger \quad$ On leave from St. Petersburg Nuclear Physics Institute, Gatchina, St. Petersburg, 188350 Russia.

[1] L. Montanet et al., Phys. Rev. D 45, 1173 (1992).

[2] G. Höhler, Pion-Nucleon Scattering, Landolt-Börnstein Vol. I/9b2 (1983), ed. H. Schopper, Springer-Verlag. We have used Karlsruhe solution KA84, generated through a FORTRAN subroutine supplied by R. Koch (private communication) [Z. Phys. C 29, 597 (1985)].

[3] R. E. Cutkosky et al., Phys. Rev. D 20, 2839 (1979); R. E. Cutkosky in Proceedings of the 4th Conference on Baryon Resonances (Toronto, 1980), ed. N. Isgur, p.19.

[4] I. Supek et al., Phys. Rev. D 47, 1762 (1993).

[5] I. V. Lopatin et al., Nucl. Phys. A567, 882 (1994).

[6] I. Sabba Stefanescu, Z. Phys. C 41, 453 (1988), and references therein.

[7] I. G. Alekseev et al., Phys. Lett. B351, 585 (1995).

[8] E. Barrelet, Nuovo Cim. 8A, 331 (1972).

[9] See, for example, the discussion in Section 2.1 of reference [2].

[10] This is clear if one considers the Goldberger-Miyazawa-Oehme sum rule [M. L. Goldberger, H. Miyazawa, and R. Oehme, Phys. Rev. 99, 986 (1955)], which relates total cross sections and scattering lengths (which are unchanged by this Barrelet transformation) to the $\pi N N$ coupling constant.

[11] R. A. Arndt et al., Phys. Rev. C 52, 2120 (1995).

[12] E. Borie, W. Gampp, G. Höhler, R. Koch, and I. Sabba Stefanescu, Z. Phys. C 4, 333 (1980). 
[13] Preliminary results using the CMB solution were reported by V. P. Kanavets, XIV International Conference on Particles and Nuclei, Williamsburg, May 1996. 


\section{Figure captions}

Figure 1. Comparison of the original and transformed $\mathrm{F}^{+}(w)$ zero-trajectories which cross the unit circle near $0.7 \mathrm{GeV}$. Energy values (in GeV units) are marked by $\mathrm{x}$ and $\mathrm{z}$ symbols on the original and transformed trajectories respectively.

Figure 2. The spin-rotation parameter $A$ for $\pi^{+} p$ elastic scattering at $1.3 \mathrm{GeV}$. The original KA84 solution (solid line) is compared to the Barrelet-transformed solution (dot-dashed line). Data are taken from Ref. [7]].

Figure 3. Contour plot of differences between the modified and original KA84 solutions (see text). The white and black regions correspond to differences less than -0.48 and greater than +1.15 respectively. Neighboring contours differ by approximately 0.2 .

Figure 4. Partial-wave amplitudes for the KA84 solution. The real (solid line) and imaginary (long dot-dashed line) parts of the original solution are compared to the real (dashed line) and imaginary (short dot-dashed line) parts of the Barrelettransformed solution. The dotted line gives the unitarity constraint $\left(\operatorname{Im} T-|T|^{2}\right)$ for the original solution. (a) $\mathrm{S}_{31}$, (b) $\mathrm{P}_{31}$, (c) $\mathrm{P}_{33}$, (d) $\mathrm{D}_{33}$, (e) $\mathrm{D}_{35}$, (f) $\mathrm{F}_{35}$, (g) $\mathrm{F}_{37},(\mathrm{~h}) \mathrm{G}_{37},(\mathrm{i}) \mathrm{G}_{39},(\mathrm{j}) \mathrm{H}_{39},(\mathrm{k}) \mathrm{I}_{311}$. 


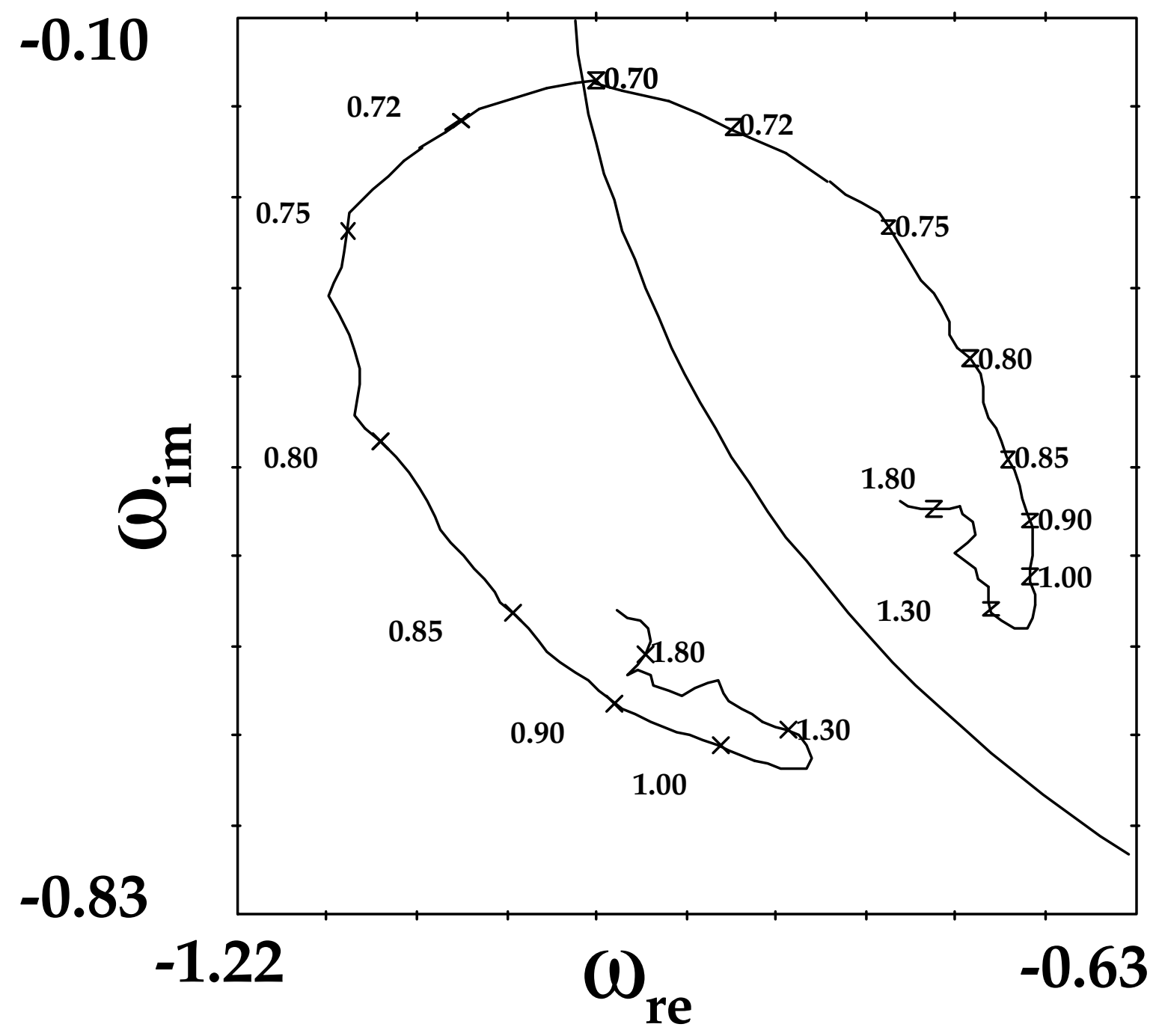




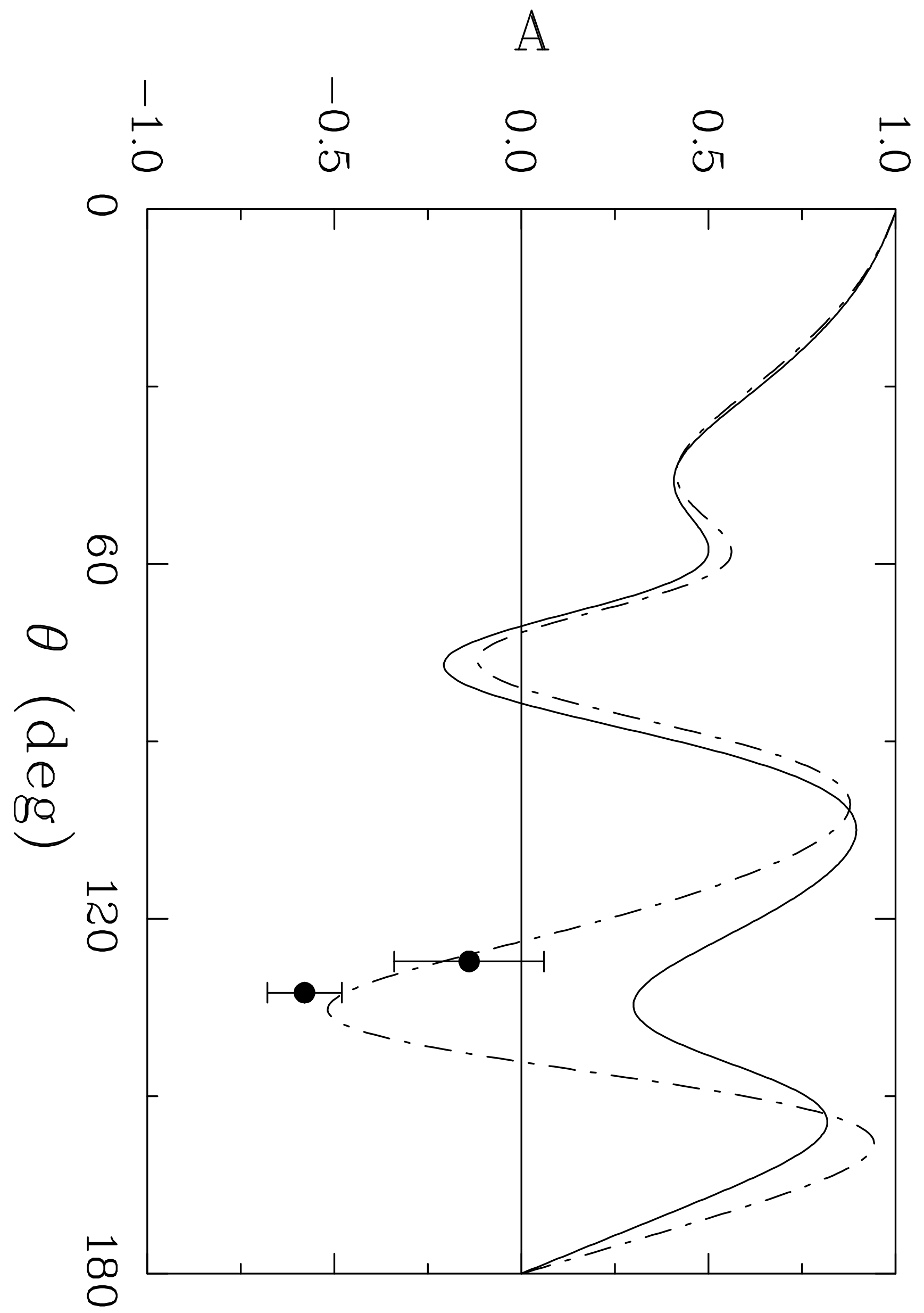




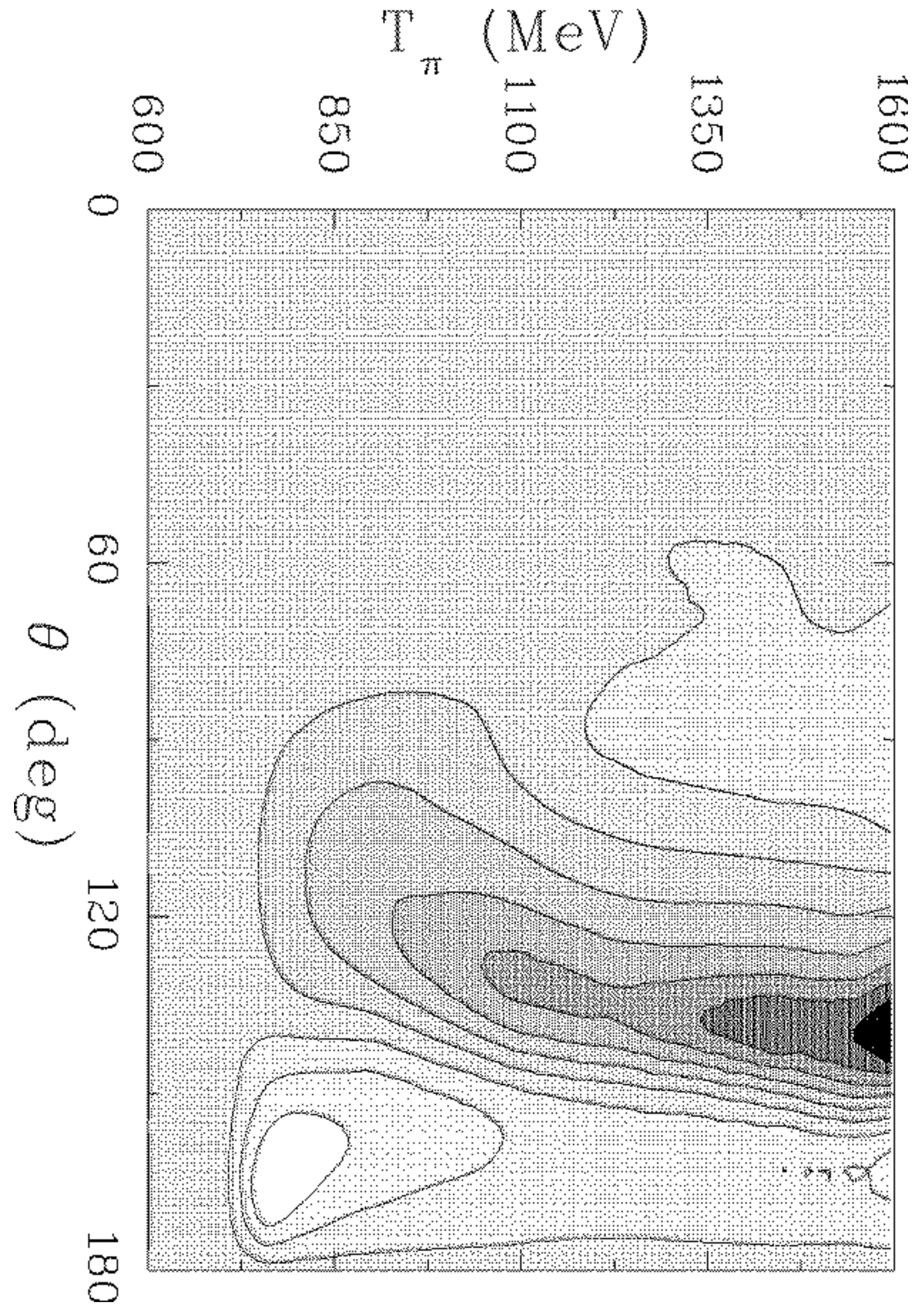




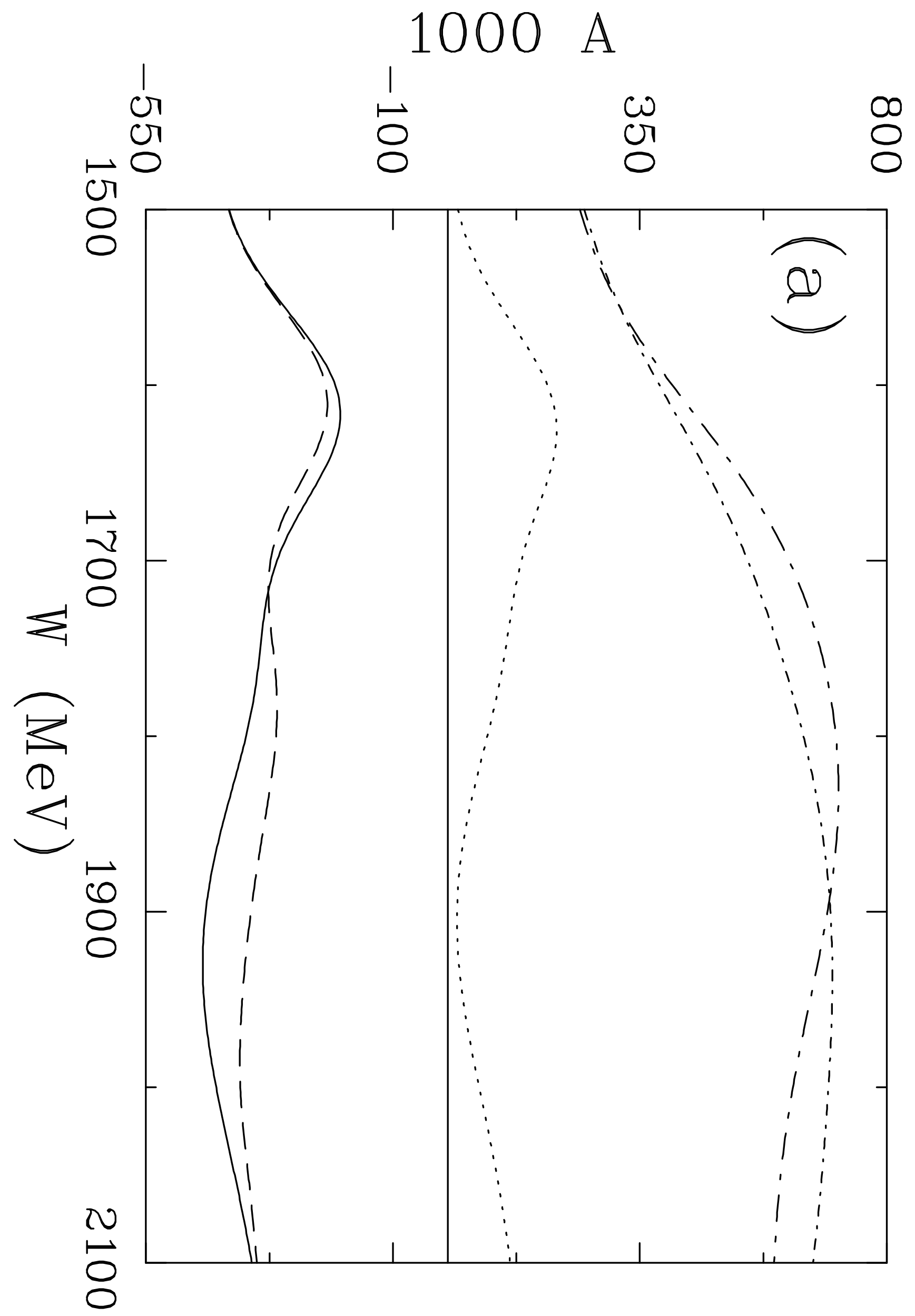









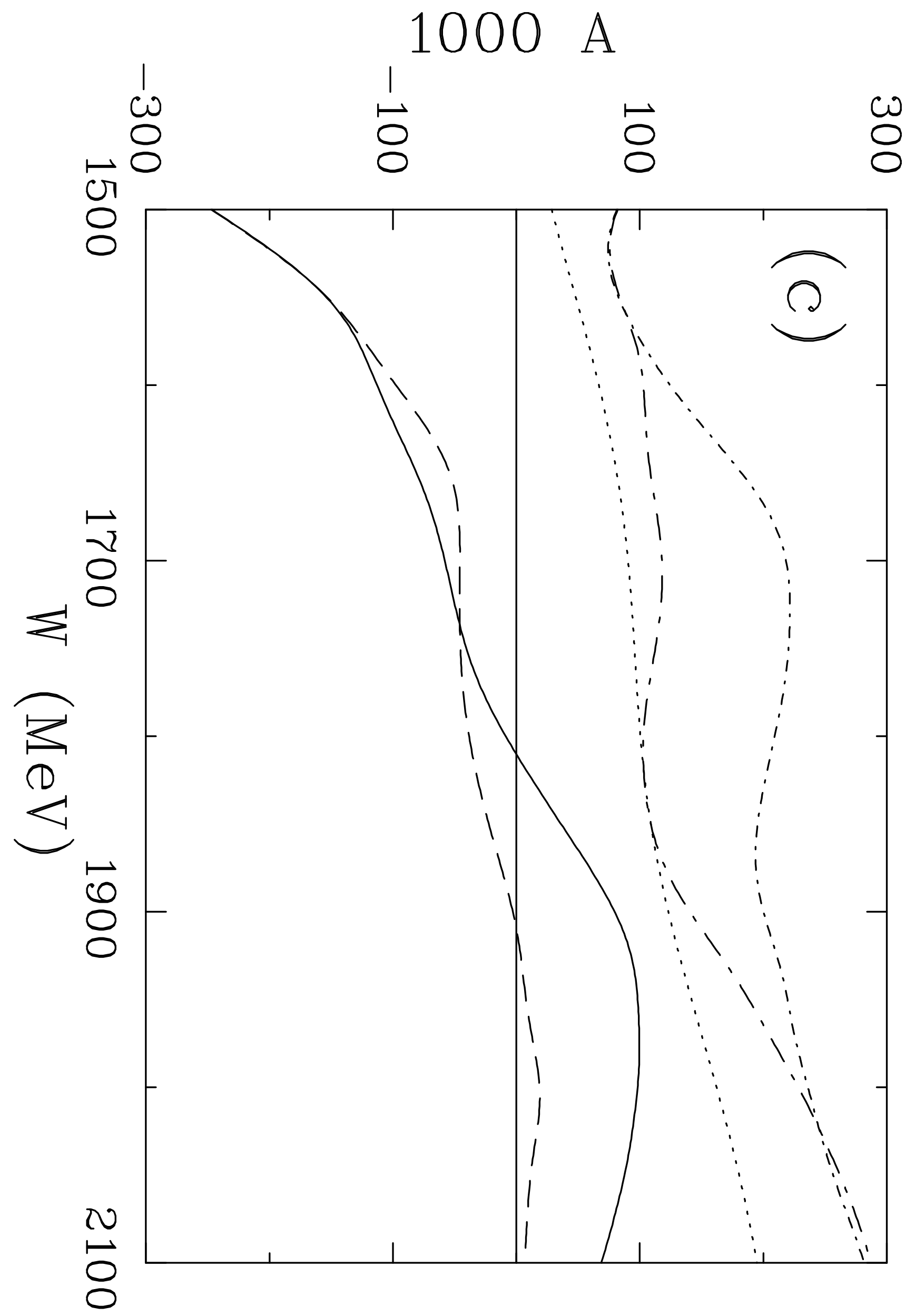




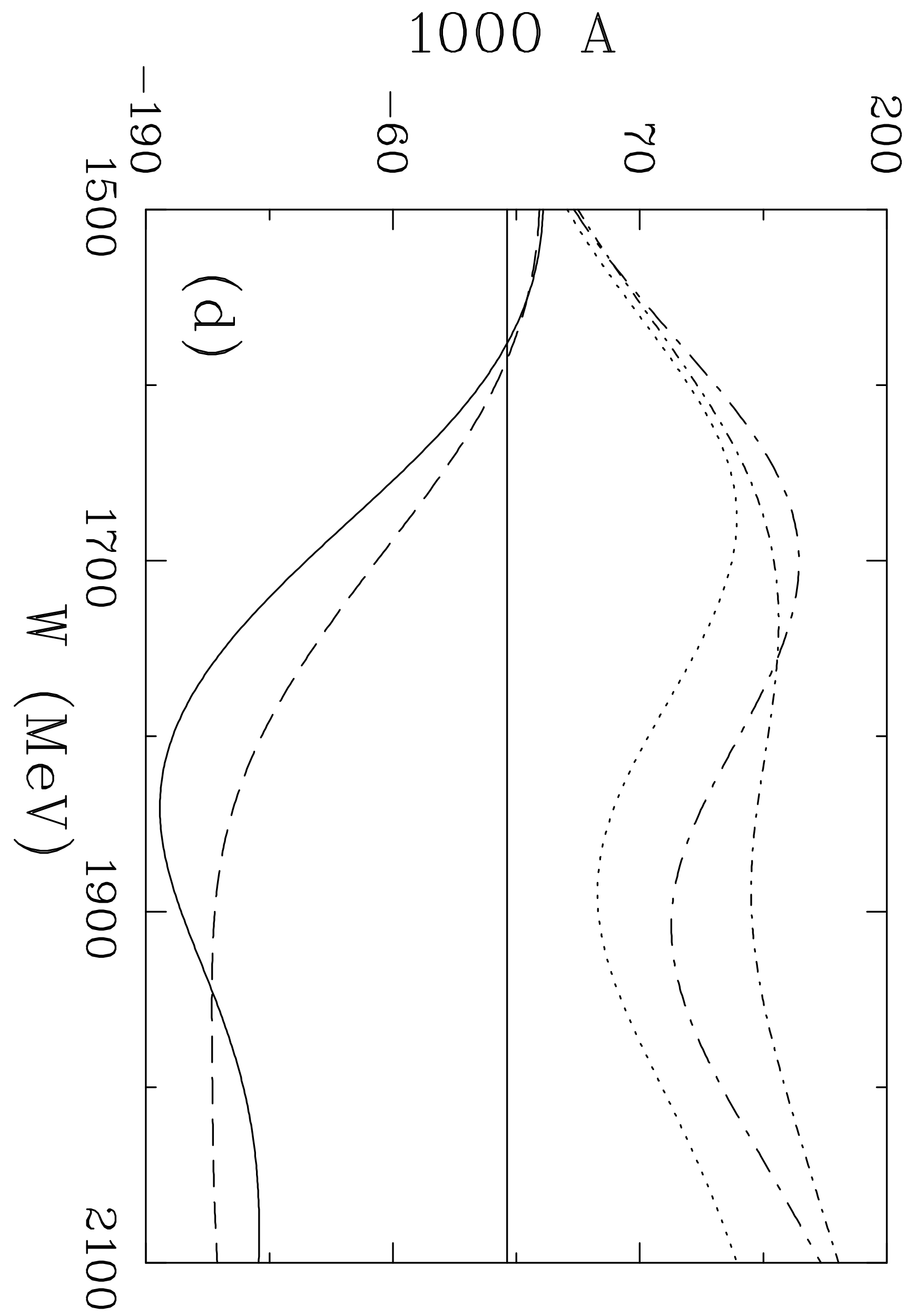




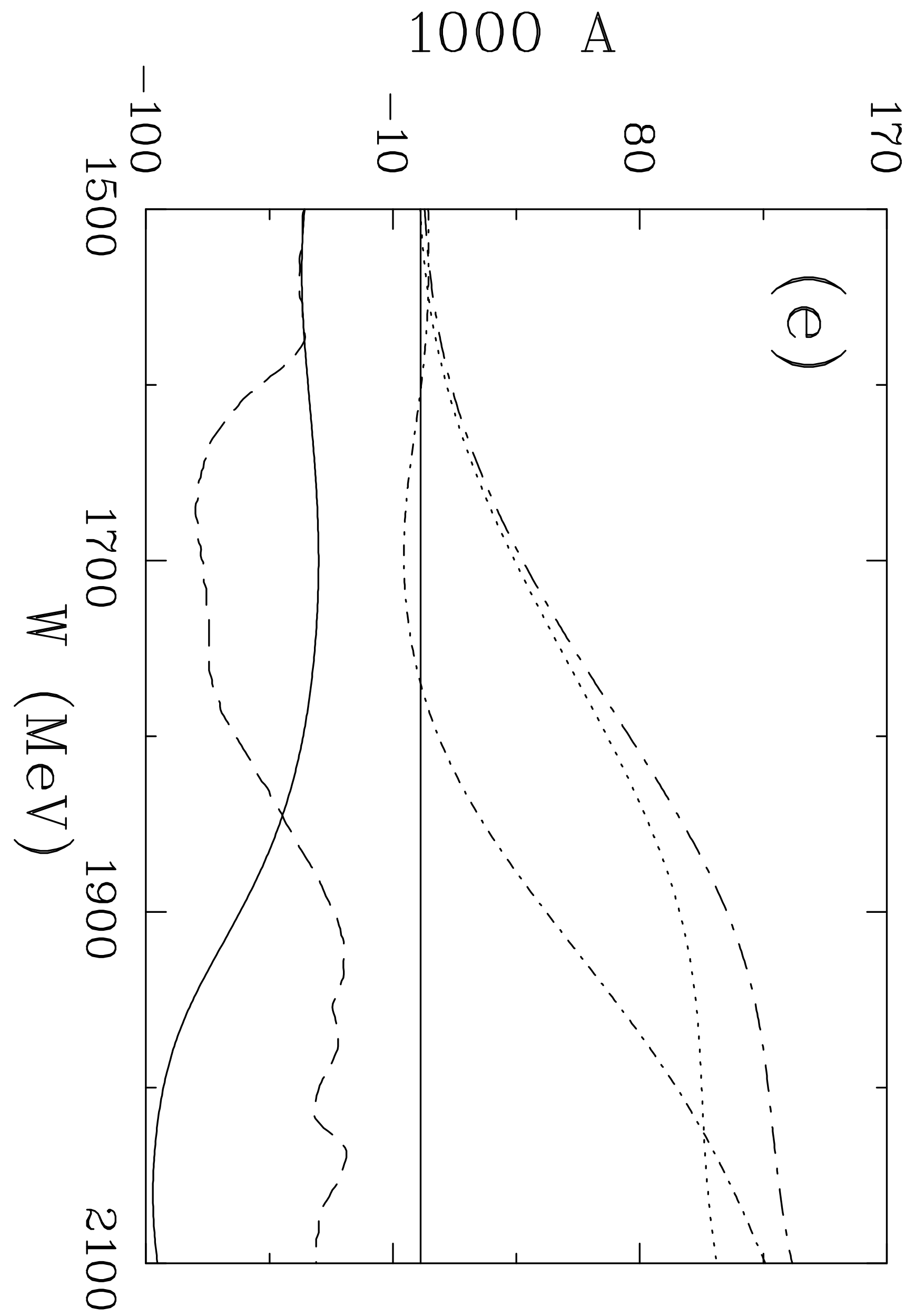




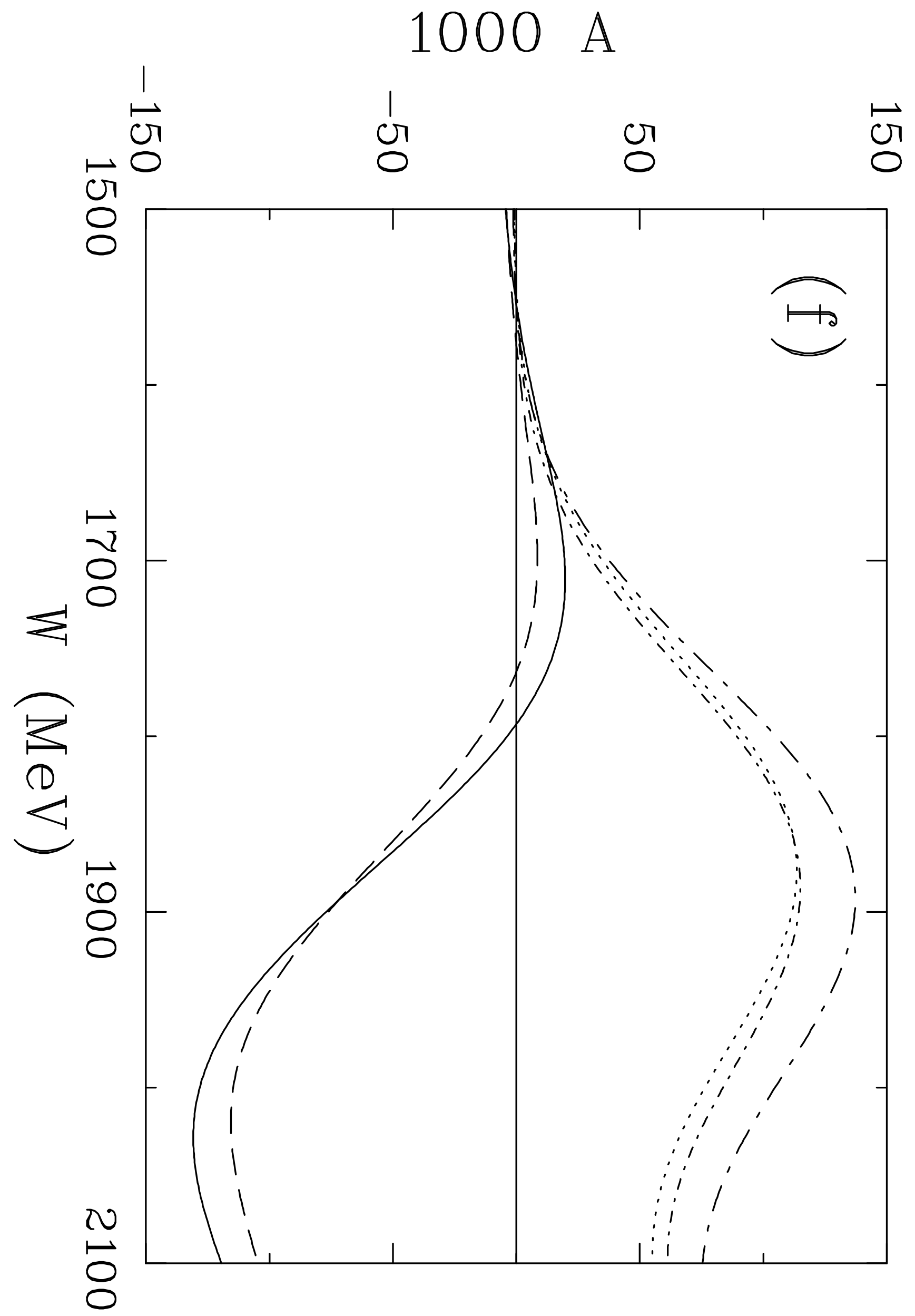




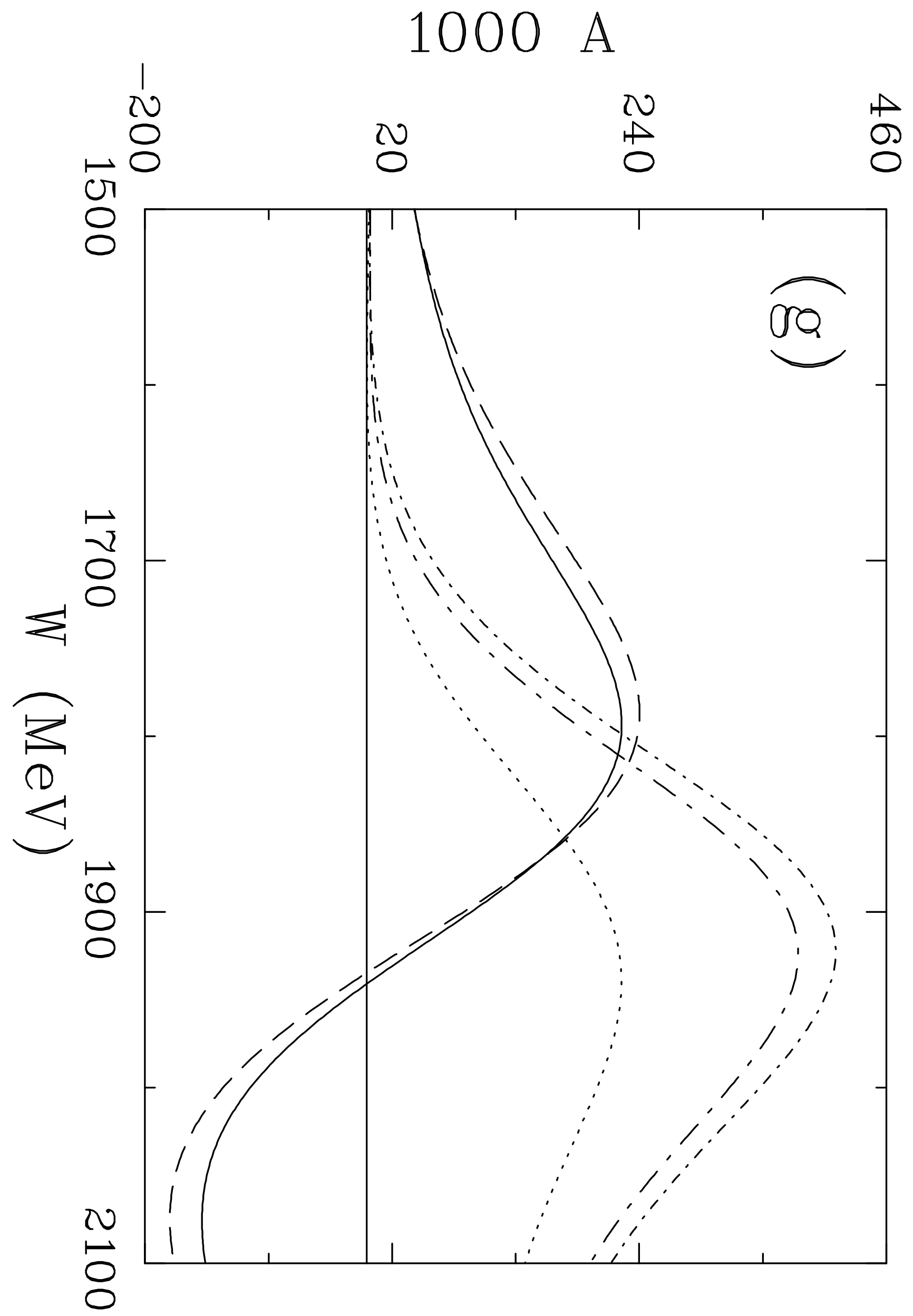




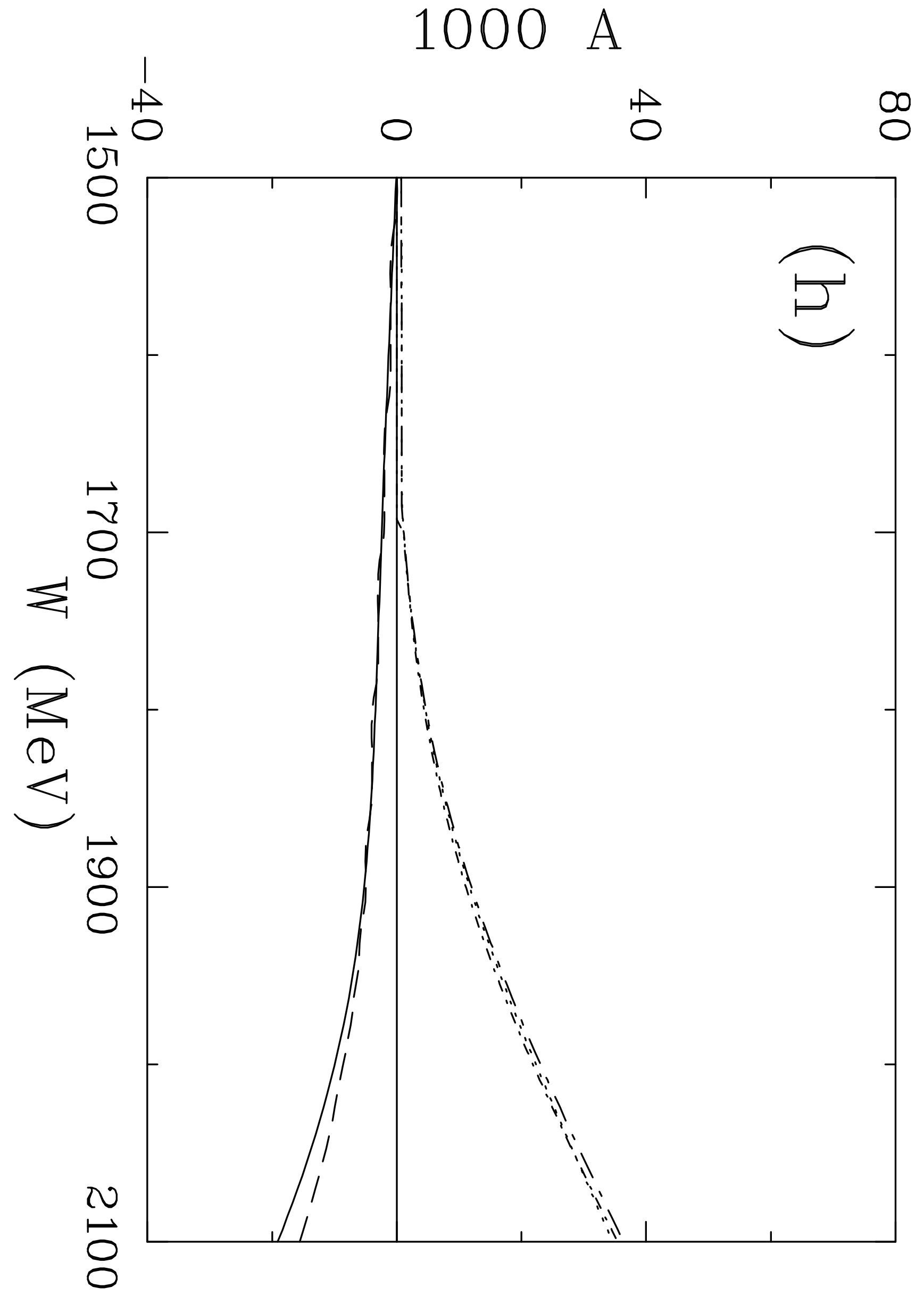




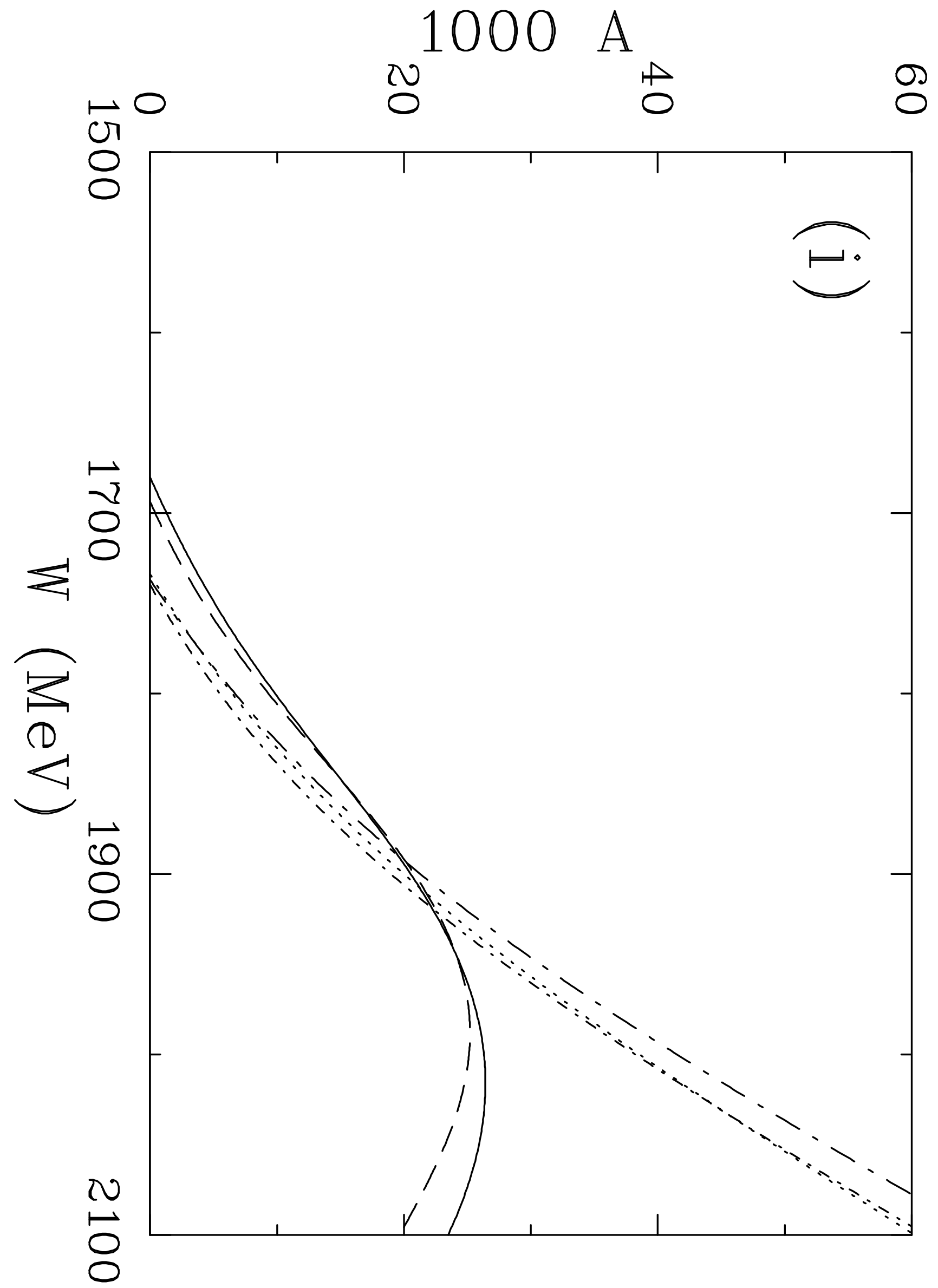




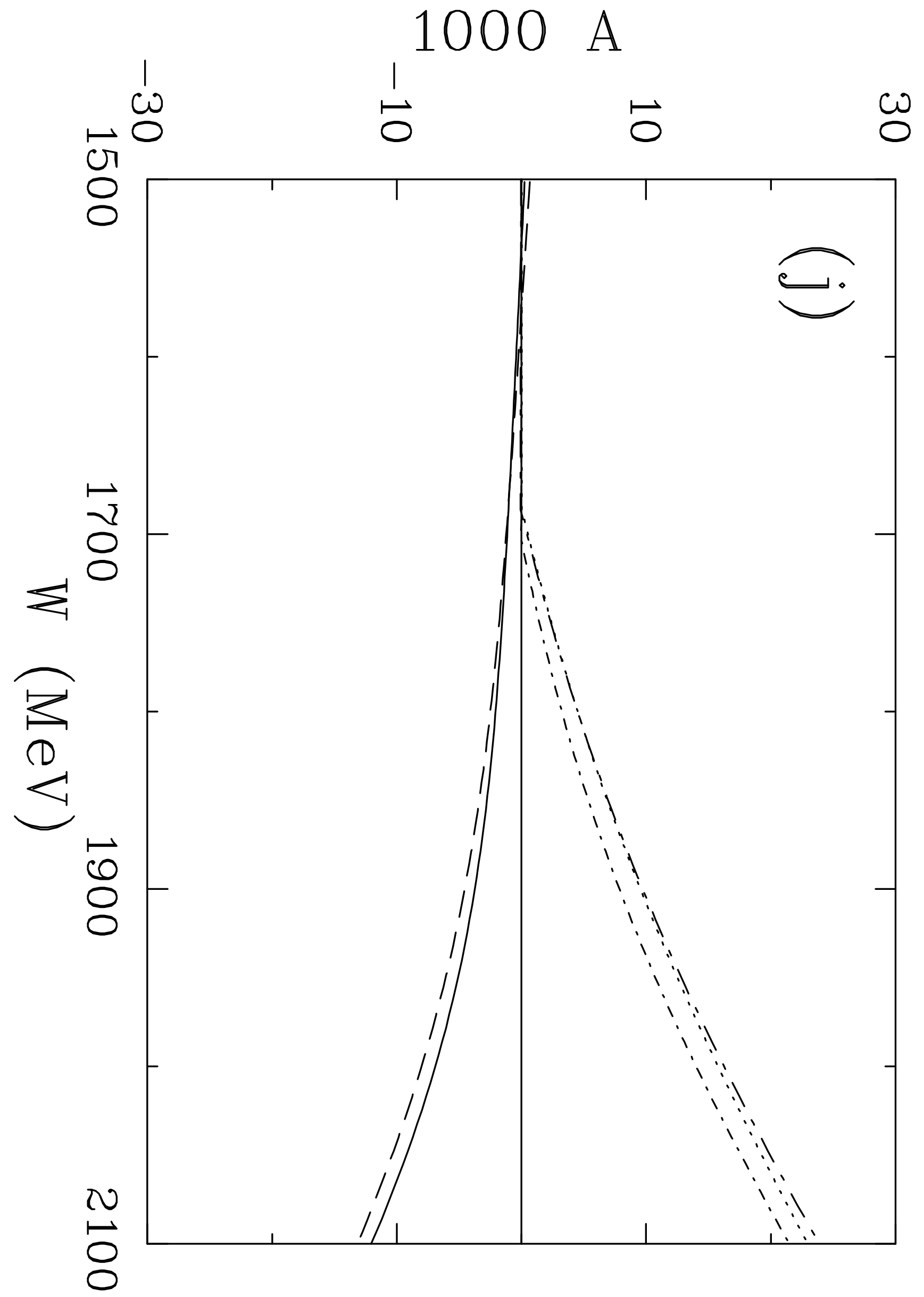




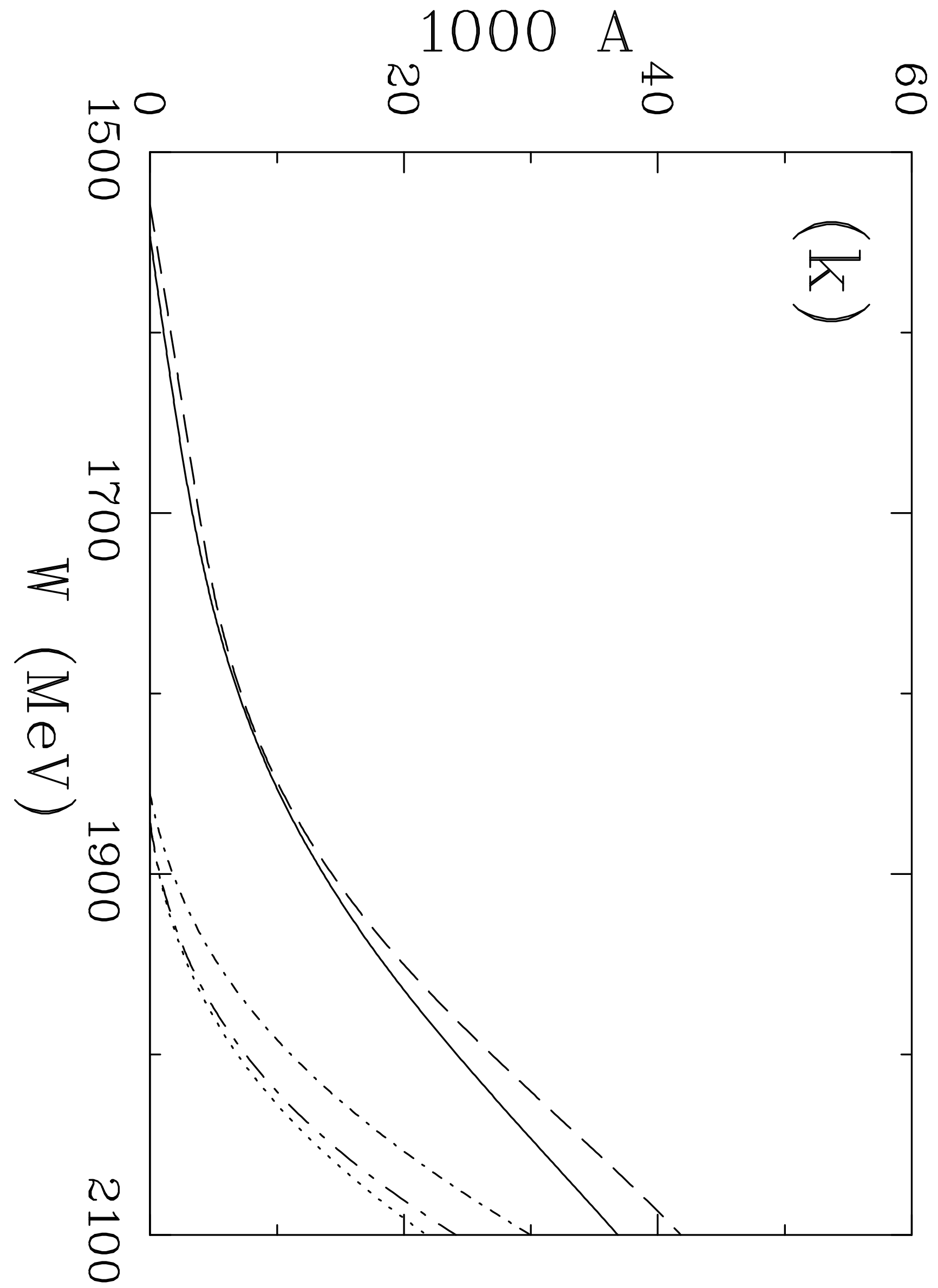

